\title{
Avaliação da Concentração de Cádmio, Cobre, Ferro, Manganês, Níquel e Zinco em Anomalocardia brasiliana (Gmelin, 1791) Provenientes de Zonas de Manguezal da Região de São Francisco do Conde e Madre de Deus, Recôncavo Baiano, BA
}

\author{
T. B. de Jesus, ${ }^{1 *}$ L. G. Fernandez ${ }^{2}$ \& A. F. De S. Queiroz ${ }^{2}$ \\ 1 Laboratório de Ciências Ambientais, Universidade Estadual do Norte Fluminense Darcy Ribeiro, Av. Alberto Lamego, 2000, \\ CEP 28015-602, Campos dos Goytacazes, RJ, Brasil \\ ${ }^{2}$ Universidade Federal da Bahia, Instituto de Ciências da Saúde, Departamento de Bio-Função, Av. Reitor Miguel Calmon, \\ $\mathrm{s} / \mathrm{n}$, Vale do Canela, CEP 40110-100, Salvador, BA, Brasil \\ (Received May 1, 2008; Accepted October 10, 2008)
}

\begin{abstract}
RESUMO
As concentrações de cádmio $(\mathrm{Cd})$, cobre $(\mathrm{Cu})$, ferro $(\mathrm{Fe})$, manganês $(\mathrm{Mn})$, níquel $(\mathrm{Ni})$ e zinco $(\mathrm{Zn})$ foram investigadas em espécimes do molusco bivalve Anomalocardia brasiliana (Gmelin, 1791), em nove estações situadas em zonas de manguezal no entorno da Baía de Todos os Santos, Bahia, Brasil. Os locais foram selecionados segundo a existência de possíveis fontes antrópicas. As análises foram realizadas através da espectrometria de absorção atômica, após secagem e digestão da amostra. As concentrações de $\mathrm{Cd}, \mathrm{Fe}, \mathrm{Mn}$ e Ni diferiram estatisticamente $(\mathrm{p}<0,05)$ nas diferentes áreas amostradas. Áreas com forte influência industrial, estação de Coqueiro Grande, nordeste da Baía de Todos os Santos, e Fazenda Cajaíba, localizada ao norte, apresentaram as maiores concentrações para os metais em estudo. Apesar das altas concentrações encontradas, nenhum dos metais analisados apresentou valores acima do limite estabelecido pela Legislação Brasileira para consumo alimentar.
\end{abstract}

Palavras-chave: metais, manguezal, Baía de Todos os Santos, molusco.

\begin{abstract}
Evaluation of the concentration the cadmium, cupper, iron, manganese, nickel and zinc in Anomalocardia brasiliana (Gmelin, 1791) distributed in mangrove areas of São Francisco do Conde and Madre de Deus, Recôncavo Baiano, BA
\end{abstract}

Cadmium $(\mathrm{Cd})$, cupper $(\mathrm{Cu})$, iron $(\mathrm{Fe})$, manganese $(\mathrm{Mn})$, nickel $(\mathrm{Ni})$ and zinc $(\mathrm{Zn})$ concentrations had been investigated in specimens of the mollusc bivalve Anomalocardia brasiliana (Gmelin, 1791), distributed at nine stations situated in mangroves zones, located at the Baía de Todos os Santos, Bahia, Brazil. Collection sites were selected based on the existence of antropic sources. The analyses had been carried through the atomic absorption spectrometry after drying and digestion of the sample. The concentrations of $\mathrm{Cd}, \mathrm{Fe}, \mathrm{Mn}$ and $\mathrm{Ni}$ had differed statistical $(\mathrm{p}<0.05)$ at the different areas. The sites with strong industry influence, Coqueiro Grande at the northeast part of the Baía de Todos os Santos, and Fazenda Cajaíba located at the northern part, had presented the highest concentrations for metals in study. Despite the high concentrations found none of the analysed metals were above the limit established of the Brazilian legislation for human consumption.

Key words: metal, cadmium, Baía de Todos os Santos, mollusc.

\footnotetext{
*Corresponding author: Taise Bomfim de Jesus, e-mail: taisebj@hotmail.com.
} 


\section{INTRODUÇÃO}

Desde a época da revolução industrial, o ambiente aquá-tico vem sendo impactado pelo aumento de descarga de metais. Em algumas áreas, a exposição de organismos vivos a poluentes orgânicos e inorgânicos causa interação entre esses agentes químicos e os sistemas biológicos, o que pode promover distúr-bios bioquímicos e/ou respostas adaptativas (Masfaraud et al., 1992).

A crescente demanda, pelo homem, por compostos e manufaturados contendo metais e produtos artefatos metálicos propriamente ditos tem causado aumento considerável na mobilização desses elementos para o meio ambiente. A atividade humana é hoje responsável por mais da metade das emissões globais de metais pesados para o meio ambiente (Nriagu, 1990).

O Brasil possui uma das maiores faixas litorâneas do mundo, cerca de $8.500 \mathrm{~km}$, incluindo diferentes ecossistemas de alto interesse biológico para a manutenção da biodiversidade e dos estoques pesqueiros, como recifes de corais e manguezais. Os manguezais ocorrem preferencialmente em áreas de baixa energia hídrica e com predominância de sedimento argilo-siltoso, características típicas de regiões estuarinas. Entretanto, exatamente nesse tipo de litoral ocorre a maior parte do desenvolvimento urbano e industrial dos países tropicais (Germen/UFBA, 1997).

A Baía de Todos os Santos, a maior baía do Brasil, com $1086 \mathrm{~km}^{2}$ (Lessa et al., 2001), constitui um grande sistema estuarino, sendo que a maior parte da sua franja interna é formada por densos manguezais (Germen/UFBA, 1997).

Atualmente, o transporte e o refinamento de petróleo na Baía de Todos os Santos são, em sua maioria, atividades indus-triais de grande porte que podem contribuir para o desequilíbrio ambiental do local. Além disso, antigas fábricas de papel locali-zadas ao norte da baía colaboraram com a presença de óleo composto por hidrocarbonetos e metais pesados, desprezados nos sistemas ambientais, como água e sedimento, sem qualquer tratamento.

Estudos anteriores de Souza et al. (1978), Tavares et al. (1979) e Wallner- Kersanach et al. (1994) relatam o impacto do óleo sobre a ecologia da Baía de Todos os Santos (CRA, 2001). Assim, o desenvolvimento de técnicas de diagnóstico ambiental e a aplicação de programas de monitoramento devem ser prio-rizados.

Invertebrados marinhos como os moluscos bivalves têm a capacidade de concentrar em seus tecidos metais tóxicos não-essenciais, como $\mathrm{Cd}, \mathrm{Ag}, \mathrm{Hg}, \mathrm{Pb}$ e $\mathrm{Cr}$, ou metais essenciais, considerados nutricionalmente importantes, como $\mathrm{Cu}, \mathrm{Zn}, \mathrm{Mn}$ e Co (Rezende \& Lacerda, 1986; Thompson et al., 1987; Szeefer et al., 1997).

A bioacumulação é definida como a transferência para os organismos de determinada quantidade de elementos químicos ou substâncias que se encontram no ambiente (Cohen, 1985). Os processos de biodisponibilidade dependerão do tipo de absorção realizada pelo organismo, do tamanho das partículas ou da fase em que os metais se encontram (dissolvido ou particulado). No caso de moluscos bivalves, estes vão absorver metais ligados à fase particulada. Sob certas condições ambientais, entretanto, esses elementos podem ser bioacumulados em concentrações tóxicas, causando danos biológicos e, consequentemente, ecológicos (Feedman, 1995). Espécies diferentes, entretanto, exibem taxas diferentes de bioacumulação, e alguns bivalves são mais apropriados como bioindicadores do que outros (Phillips et al., 1982, Phillips \& Segar, 1986). Em estudos realizados por Peso-Aguiar et al. (1991), a $A$. brasiliana demons-trou habilidade indicadora, revelando, de modo significativo, o grau de contaminação nos diferentes pontos a partir de uma fonte poluidora.

O molusco comestível $A$. brasiliana é consumido pela população dos municípios de São Francisco do Conde, Madre de Deus e suas cercanias, todos inseridos na Baía de Todos os Santos. A contaminação do alimento marinho pode condizer com um prejuízo local, caso o estudo aponte uma fonte de metal existente na área.

O objetivo deste trabalho é avaliar o grau de contaminação por metais pesados $(\mathrm{Cd}, \mathrm{Cu}, \mathrm{Fe}, \mathrm{Mn}, \mathrm{Ni}$ e $\mathrm{Zn})$ na área, comparando com estudos anteriores, e verificar os possíveis riscos da exposição ao metais associados ao molusco $A$. brasiliana pela população em geral.

\section{MATERIAL E MÉTODOS}

\section{Areas de estudos}

A Baía de Todos os Santos (Lat. $13^{\circ} \mathrm{S}-$ Long. $38^{\circ} 35^{\prime} \mathrm{W}$ ) está localizada no nordeste da costa brasileira. Inserida no Recôn-cavo Baiano, esta baía é intensamente povoada por pequenas cidades da Região Metropolitana de Salvador (RMS) e da capital do Estado, na sua maior extensão (Germen/UFBA, 1997). Os rios Paraguaçu e Subaé são os principais afluentes da baía. A maior ilha, Itaparica, é separada do continente pelo estreito canal de Itaparica.

Atualmente, os recursos oriundos das atividades petrolíferas são as principais fontes de captação de renda da econo-mia local, além da produção de itens agrícolas e da atividade pesqueira, de menor relevância financeira para a região. A atividade pesqueira, porém, é mais afetada pelos riscos poten-cias de contaminação que são gerados pela atividade industrial, que afetam a qualidade dos ecossistemas dessa região, ofere-cendo riscos potenciais aos organismos e à população local que depende dos recursos pesqueiros como fonte de renda e alimentação.

Foram escolhidas nove estações amostrais distribuídas em manguezais localizados ao norte da Baía de Todos os Santos, sendo definidas cinco estações nos manguezais do município de São Francisco do Conde: estação 1: Coqueiro Grande (S 12 $43^{\circ} 00^{\prime}$ ' W

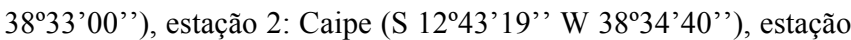
5: Ilha de Fontes (S 12 $40^{\prime} 16^{\prime \prime}$ W 38 39'15'), estação 6: Fazenda Cajaíba (S 12 40'14', W 38 41'04') e estação 7: Ilha do Pati (S 1242'31' W 38 37'20' '); duas em Madre de Deus: estação 3: Suape ( S 1243'02'" W 38 34'40') e estação 4: Fábrica de Asfalto ( S 1243'57" W 38 37'21"); e uma no município de Saubara: a estação 8: Cabuçu ( S 12 49' 41', W 
$38^{\circ} 55^{\prime}$ 10') (Figura 1). Foi estabelecida uma estação de referên-cia na zona de manguezal do município da Ilha de Vera Cruz (Ilha de Itaparica), estação 9 ou estação de referência Jeribatuba, por estar geograficamente posicionada em uma área distante da influência de atividades petrolíferas.

\section{Metodologia}

A espécie em estudo é um organismo filtrador, habita zonas entre-marés de baía e enseadas abrigadas, de águas rasas com profundidade variando entre $0,5 \mathrm{~m}$ e $1,5 \mathrm{~m}$ (Moueza et al., 1999), e vive enterrada em sedimento arenoso e areno-lodoso a uma profundidade de $5 \mathrm{~cm}$ do substrato com distribuições do tipo agregada (Wallner-Kersanach et al., 1994).

Durante a coleta dos moluscos em estudo foram medidos na água superficial os seguintes parâmetros: $\mathrm{pH}$ (phmetro Portátil Hanna Instruments, modelo HI 8314), oxigênio dissolvido (oxímetro portátil Hanna Instruments, modelo HI 9146), salini-dade (refratômetro Salinity Refractometer S-1 Shibuya Optical Co. Ltda.) e temperatura (oxímetro), de todas as estações, nas proximidades do local onde se efetivava a coleta das amostras biológicas.

As amostras foram coletadas em julho de 2003 e fevereiro de 2004. A escolha dos indivíduos amostrados foi aleatória por toda estação amostral. A faixa de tamanho dos moluscos coleta-dos variou de 15,16 a 28,94 mm. Em cada estação amostral foram coletados 30 indivíduos e preparado um "pool” (amostra composta) para reduzir a variação individual na concentração de metais pesados (Daskalakis, 1996; Szefer et al., 1997). Depois da lavagem dos espécimes, para a retirada do sedimento aderido nas conchas, as partes moles foram retiradas, homogeneizadas e colocadas na estufa a $100^{\circ} \mathrm{C}$ por 12 horas, para a obtenção do peso seco. As amostras, analisadas em triplicata, sofreram digestão ácida por micro-ondas $\left(\mathrm{HNO}_{2}\right.$ Suprapur) (Camusso et al., 2001). Essa técnica é menos complicada e consome menor tempo que o método de dissolução convencional. Após a digestão, foram analisados seis elementos $(\mathrm{Cd}, \mathrm{Cu}, \mathrm{Fe}, \mathrm{Mn}, \mathrm{Ni}$ e $\mathrm{Zn})$, através de Espectrometria de Absorção Atômica com Chama, utilizando equipamento, marca VARIAN, modelo AA 800. Os metais foram selecionados com base no estudo de Förstner (1993), que sugere, entre outros, $\mathrm{Cd}, \mathrm{Cu}, \mathrm{Fe}, \mathrm{Mn}, \mathrm{Ni}$ e $\mathrm{Zn}$ como provenientes de resíduos sólidos oriundos de refinarias.

Para a comparação das concentrações de metais encon-tradas neste estudo com os valores máximos de metais estabe-lecidos pela ANVISA (1998), foi calculado o peso úmido. O fator de conversão utilizado foi a média da porcentagem de umidade dos organismos nas diferentes áreas coletadas, que aponta $86 \%$ de teor de umidade para a A. brasiliana, desta forma, devem-se multiplicar os valores de metais obtidos para o peso seco por 0,14 a fim de se obterem os valores para peso úmido.

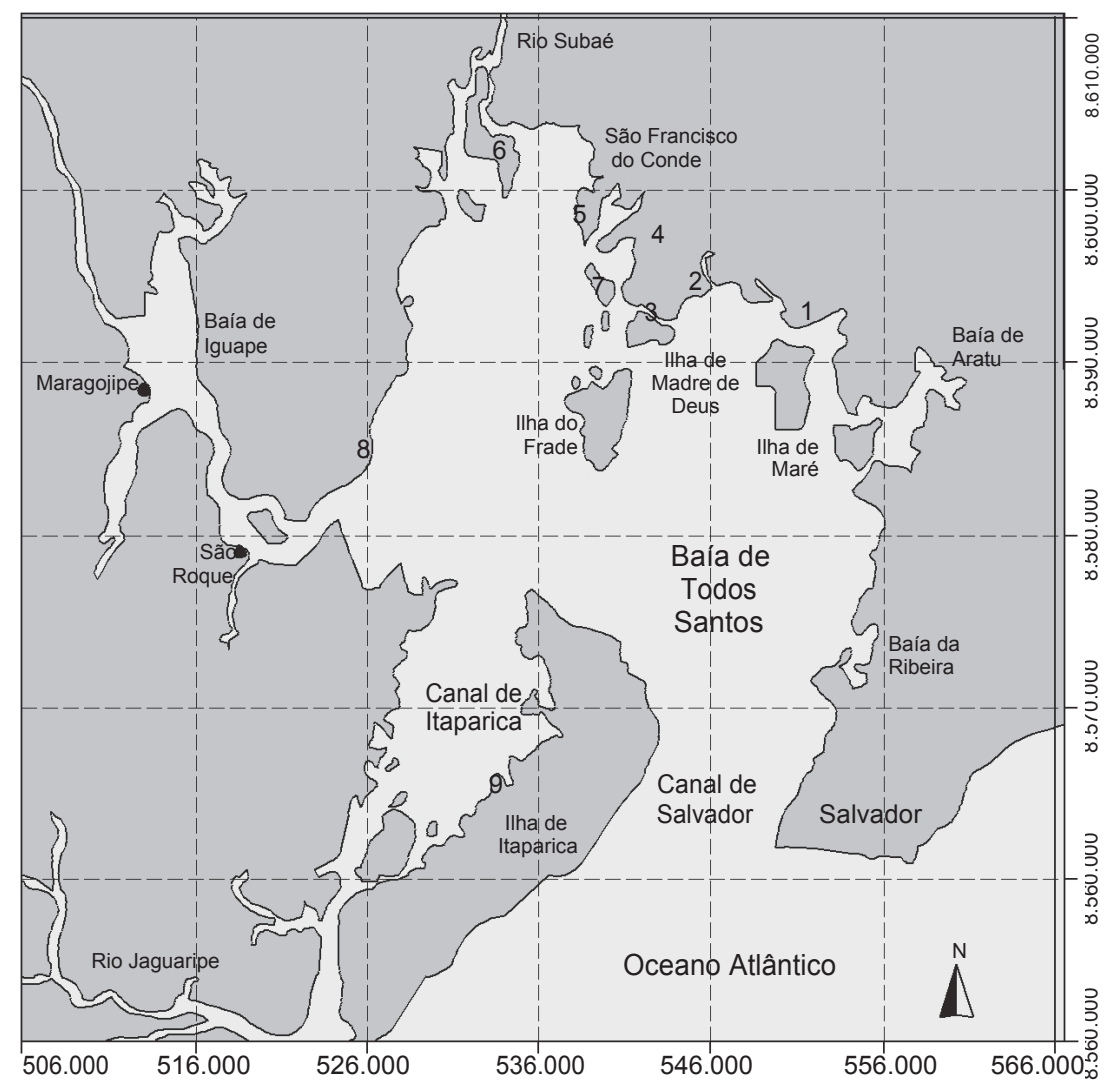

Figura 1 - Localização das áreas de estudo distribuídas em manguezais da Baía de Todos os Santos, Bahia, Brasil. 


\section{Controle analítico do método}

O ácido nítrico usado para a digestão foi Suprapur (Merck). As soluções estoques (Merck) de $1000 \mathrm{mg} \mathrm{L}^{-1}$ para diferentes elementos analisados foram empregadas no preparo do standard de calibração.

A digestão e o procedimento analítico foram checados através da análise do material de referência de tecido de ostras (SRM-1566b, NIST, USA) em todas as digestões. As concen-trações dos metais quantificados no material de referência certificado se encontram na Tabela 1 .

Foram realizadas análises de replicatas das amostras durante toda a digestão. O potencial de contaminação das amostras foi determinado analisando-se amostras contendo apenas o ácido (branco) em toda a digestão.

\section{Tratamento estatístico}

Como os dados referentes às concentrações dos metais analisados, tanto originais quanto transformados por logaritimização na base natural, não são normais, desta forma, aplicou-se, para cada metal, a alternativa não paramétrica, nomeadamente, a ANOVA não-paramétrica de Friedman (Zar, 1996). A distribuição não-paramétrica é geralmente atribuída, nesses estudos, às variações individuais na entrada e à excreção de metais pesados pelo organismo (Lobel et al., 1982) causada pelas diferenças fisiológicas e metabólicas entre orga-nismos.

Toda a análise estatística foi realizada com o auxílio do programa STATISTIC, v. 6,0. Para comparar a concentração total de metais em diferentes estações amostrais, o índice de poluição do metal (MPI) foi usado, obtido com a equação (Usero et al., 1997):

$$
\mathrm{MPI}=(\mathrm{Cf}, \cdot \mathrm{Cf}, \ldots \mathrm{Cfn},)^{1 / \mathrm{n}}
$$

em que $\mathrm{Cfi}=$ concentração do metal $\mathrm{n}$ nas diferentes áreas amostrais.

\section{RESULTADOS}

A média dos parâmetros físico-químicos das estações amostradas apresentados na Tabela 2 demonstram um valor baixo de oxigênio dissolvido, de $3,4 \mathrm{mg} \mathrm{L}^{-1}$, em Coqueiro Grande (estação 1), quando comparada com as outras estações amos-trais. Em relação ao pH, todas as estações apresentaram caráter básico, variando de 7,77 na estação de Fábrica de Asfalto a 8,07 na Ilha do Pati (estação 7).

Tabela 1 - Concentração média de metais $\left(\mu \mathrm{g} \mathrm{g}^{-1}\right.$ peso seco) nas amostras de referência de tecidos de ostras (SRM 1566b, NIST, USA), comparando-as com as concentrações analisadas $(n=4)$.

\begin{tabular}{ccc}
\hline & Concentrações certificadas & Concentrações analisadas \\
\hline $\mathrm{Cu}$ & $71,6 \pm 1,6$ & $64,3 \pm 2$ \\
$\mathrm{Mn}$ & $18,5 \pm 0,2$ & $18,5 \pm 0,08$ \\
$\mathrm{Fe}$ & $205,8 \pm 6,8$ & $190,35 \pm 13,52$ \\
$\mathrm{Ni}$ & $1,04 \pm 0,09$ & $1,68 \pm 0,61$ \\
$\mathrm{Cd}$ & $2,48 \pm 0,08$ & $2,50 \pm 0,6$ \\
$\mathrm{Zn}$ & $1424 \pm 46$ & $1899 \pm 161$ \\
\hline
\end{tabular}

Tabela 2 - Média e desvio-padrão dos parâmetros físico-químicos coletados na água superficial das estações amostrais.

\begin{tabular}{|c|c|c|c|c|}
\hline Estações amostrais & $\mathbf{p H}$ & $\begin{array}{c}\text { Temperatura } \\
\left({ }^{\circ} \mathrm{C}\right) \\
\end{array}$ & $\begin{array}{c}\text { Oxigênio } \\
\text { dissolvido } \\
\left(\mathrm{mg} \mathrm{L}^{-1}\right)\end{array}$ & Salinidade \\
\hline 1- Coqueiro Grande & $7,95 \pm 0,03$ & $25,5 \pm 0,49$ & $3,43 \pm 1,64$ & $37,3 \pm 1,55$ \\
\hline 2- Caípe & $7,97 \pm 0,03$ & $28,4 \pm 2,61$ & $4,22 \pm 0,26$ & $37,5 \pm 2,47$ \\
\hline 3-Suape & $8,04 \pm 0,26$ & $29,9 \pm 3,46$ & $5,96 \pm 1,83$ & $38,7 \pm 2,61$ \\
\hline 4- Fábrica de Asfalto & $7,77 \pm 0,60$ & $28,7 \pm 2,68$ & $5,38 \pm 0,12$ & $35,9 \pm 1,34$ \\
\hline 5- Ilha das Fontes & $7,93 \pm 0,14$ & $28,7 \pm 2,54$ & $4,74 \pm 0,30$ & $36,4 \pm 1,69$ \\
\hline 6- Fazenda Cajaíba & $8,03 \pm 0,22$ & $28,6 \pm 2,68$ & $4,87 \pm 0,007$ & $36,6 \pm 3,2$ \\
\hline 7- Ilha do Pati & $8,07 \pm 0,25$ & $27,9 \pm 2,33$ & $4,55 \pm 0,65$ & $36,1 \pm 1,48$ \\
\hline 8- Cabuçu & $7,91 \pm 0,28$ & $27,8 \pm 1,06$ & $5,26 \pm 0,92$ & $32,9 \pm 1,34$ \\
\hline 9- Jeribatuba & $7,85 \pm 0,07$ & $26,6 \pm 0,77$ & $4,88 \pm 0,25$ & $36 \pm 0,70$ \\
\hline
\end{tabular}


As concentrações da maioria dos metais no bivalve em estudo variaram notavelmente em relação às estações amostradas (Tabela 3). A ordem de acumulação dos metais estudados foi: Fé $>\mathrm{Mn}>\mathrm{Zn}>\mathrm{Cu}>\mathrm{Ni}>\mathrm{Cd}$. Nas estações 5, 6, 8 e 9, a concentração de $\mathrm{Zn}$ foi maior que a concentração de $\mathrm{Mn}$.

Com exceção de $\mathrm{Zn}$ e $\mathrm{Cu}$, os metais analisados foram significativamente diferentes $(\mathrm{p}<0,05)$ entre as áreas de estudo (Tabela 3). Fe, Mn e Ni foram os elementos que apresentaram as maiores diferenças significativas entre as áreas estudadas.

Através do cálculo do índice de poluição do metal (MPI) nas diferentes estações amostrais, é possível determinar a ordem crescente de poluição dos metais analisados $(6>1>4>$ $5>7>8>3>9>2$ ). Na estação da Fazenda Cajaíba, o MPI foi o mais elevado (38), seguido das estações de Coqueiro Grande $(29,85)$ e Fábrica de Asfalto $(28,49)$. Nas estações de Ilha das Fontes (27,33), Cabuçu $(26,4)$ e Suape $(26,26)$, os índices foram similares. A estação de Caípe obteve um índice ainda menor que Jeribatuba, estação adotada como referência (19 e 21, respectivamente).

\section{DISCUSSÃO}

caráter básico do pH nas estações demonstra midade com o mar, pela ação do tamponamento das águas estuarinas (Aragon et al., 1986). O valor baixo de oxigênio dissolvido observado na estação de Coqueiro Grande reflete os altos valores encontrados para $\mathrm{Mn}$, pois, de acordo com estudos realizados por Rezende \& Lacerda (1986), a baixa concentração de oxigênio dissolvido possibilita maior solubilidade do $1,4 \pm 0,07 \quad 8,9 \pm 0,65 \quad 233,2 \pm 22,69^{* *}$

in

A. brasiliana como biomonitor.

Tabela 3 - Concentração média de metais pesados $\left(\mu \mathrm{g} \mathrm{g}^{-1}\right.$

peso seco), em A. brasiliana, nas diferentes estações amostrais. na literatura para a concentração de metais em $A$. brasiliana.

$\mathrm{O} \mathrm{Ni}$ é o único metal que não possui concentrações de referência em A.brasiliana.

Ao comparar os valores máximos de metais encontrados neste trabalho em $A$. brasiliana com os valores descritos por Wallner-Kersanach (1994) na mesma região de estudo, nota-

se que o $\mathrm{Cd}$, o $\mathrm{Cu}$ e o $\mathrm{Zn}$ apresentaram concentrações mais elevadas. A carência de depuração dos organismos em questão ao chegar ao laboratório pode contribuir para a elevação desses metais, pois estudos de Wallner-Kersanach et al. (1994) indicam que ocorre aumento significativo de metais em $A$. brasiliana não depurada em laboratório antes da análise de metais, porém, em estudos realizados por Garcia et al. (2007) em sedimentos coletados nas mesmas áreas e no mesmo período deste trabalho,

foi relatado o aumento da concentração de $\mathrm{Cd}$, e a característica de ser filtrador permite que esse organismo reflita rapidamente a variação do poluente no meio (Coimbra, 2003).

Os valores encontrados para $\mathrm{Cu}$ neste estudo podem ser considerados elevados $\left(8,9\right.$ a $\left.19,7 \propto \mathrm{g} \mathrm{g}^{-1}\right)$ quando comparados com outros trabalhos que estudaram $A$. brasiliana, como Coimbra (2003) ( $1,65 \propto \mathrm{g} \mathrm{g}^{-1}$ a 7,6 $\left.\propto \mathrm{g} \mathrm{g}^{-1}\right)$ e Lacerda (1983) (até res con detectadas no entorno da RLAM e no rio Mataripe (Caípe Coqueiro Grande), possivelmente resultantes de uso de algicidas ... em refrigeraçao com agua salgada e da entrada de esgotos

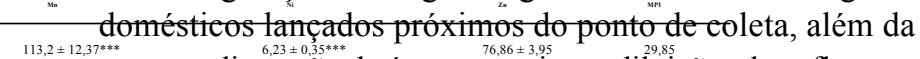

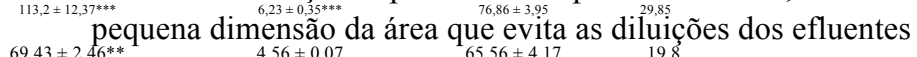

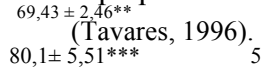
$\begin{array}{ll}6,16 \pm 0,42 \quad 71,63 \pm 9,12 & 28,49\end{array}$ $48,3 \pm 9,09^{* *} \quad 6,86 \pm 0,35^{*} \quad 80,9 \pm 2,47 \quad 27,33$ $104,6 \pm 13,50 \quad 9,76 \pm 1,9 \quad$ gênicas colaboram significativamente para um acréscimo nas $108,73 \pm 19,16 \quad 38,22$ $77,76 \pm 2,44 * * * \quad 4,93 \pm 0,28 \quad \begin{gathered}\text { concentraçôes de } \\ 74,5 \pm 5,58\end{gathered} \quad 24,92$-1 Coroa45,71 $11,12 * *$ mínima e máxima, $6,79 \pm 0,44 * * *$ respectiv $, 9 \pm 13,74 * *$ encontradas 5,16emtrabalhos $\pm 0,42$ anteriores $77,7 \pm 2,12$ variaram $21 \mathrm{de} 15 \propto \mathrm{gg}^{-1}$ a 106 $\propto \mathrm{g} \mathrm{g}^{-1}$ (Coimbra, 2003), medidas em peso seco para A. brasiliana. 
Tabela 4 - Concentração máxima de metais analisados ( $\propto \mathrm{g} \mathrm{g}^{-1}$, peso seco) em A. brasiliana comparando o presente estudo com diferentes localidades do Brasil.

\begin{tabular}{|c|c|c|c|c|c|c|}
\hline Local & Cd & $\mathbf{C u}$ & $\mathrm{Fe}$ & Mn & $\mathbf{N i}$ & $\mathbf{Z n}$ \\
\hline Presente estudo & 4,06 & 19,07 & 715,55 & 113,2 & 9,76 & 108,73 \\
\hline $\begin{array}{l}\text { Baía de Todos os Santos, BA } \\
\text { (Wallner-Kersanach, 1994) }\end{array}$ & 3 & 8,31 & - & - & - & 65,55 \\
\hline Coroa Grande, RJ (Coimbra, 2003) & - & 27,6 & 314 & 173 & - & 175 \\
\hline $\begin{array}{l}\text { Enseada das Garças, RJ } \\
\text { (Coimbra, 2003) }\end{array}$ & - & 18,3 & 381 & 183 & - & 48,5 \\
\hline $\begin{array}{l}\text { Baía de Sepetiba, RJ } \\
\text { (Lacerda, 1983) }\end{array}$ & - & 0,76 & - & 4,15 & - & 19,5 \\
\hline $\begin{array}{l}\text { Baía de Sepetiba, RJ } \\
\text { (Carvalho, 1991) }\end{array}$ & - & 5 & - & 55 & - & 84 \\
\hline
\end{tabular}

Em ostras e mariscos, as concentrações de Fe variaram de $226,0 \propto \mathrm{g} \mathrm{g}^{-1}$ a $567,0 \propto \mathrm{g} \mathrm{g}^{-1}$ (Rezende \& Lacerda, 1986) e $265,0 \propto \mathrm{g} \mathrm{g}^{-1}$ (Szefer et al., 1997). Desta forma, pode-se observar grande variação na concentração desse elemento independente da capacidade de bioacumulação do organismo, mas dependente da área de estudo.

Não há trabalhos quantificando a concentração de $\mathrm{Fe}$ na A. brasiliana da região da Baía de Todos os Santos. Os dados obtidos no presente estudo, quando comparados com os do trabalho de Coimbra (2003), em A. brasiliana, ressaltam a elevada concentração desse metal em determinadas estações amostrais (Coqueiro Grande e Ilha das Fontes).

As altas concentrações de Fe na estação de Coqueiro Grande foram responsáveis pelo alto índice de poluição do metal (MPI). Elevadas concentrações desse elemento em sedimento na região já foram observadas por Santos (2002), que sugeriu a existência de poços de perfuração, hoje já desativados, como um passivo ambiental, além de estruturas metálicas provenientes desses poços abandonados que podem estar liberando, além do $\mathrm{Fe}$, outros cátions, a exemplo do $\mathrm{Cu}$ e do $\mathrm{Zn}$.

Os valores médios obtidos para Ni no presente estudo foram semelhantes aos valores médios obtidos por Carvalho et al. (2001), Carvalho et al. (1993) e Rezende \& Lacerda (1986) $\left(8,9 \propto \mathrm{g} \mathrm{g}^{-1}, 7,4 \propto \mathrm{g} \mathrm{g}^{-1} \mathrm{e} 12,1 \propto \mathrm{g} \mathrm{g}^{-1}\right.$, respectivamente). Todos os estudos citados utilizaram o Perna perna (Linnaeus, 1758) como biomonitor, em áreas ditas como naturais ou pouco contami-nadas por metais pesados.

As concentrações médias de $\mathrm{Zn}$ encontradas neste estu-do $\left(79,3 \propto \mathrm{g} \mathrm{g}^{-1}\right)$ foram semelhantes às obtidas por Wallner-Kersanach (1994) na Baía de Todos os Santos. Valores mais elevados de Zn em A. brasiliana foram encontrados por Coimbra (2003) (175 $\propto \mathrm{g}$ $\mathrm{g}^{-1}$ ) na Baía de Sepetiba. Esse autor considerou a concentração obtida normal para o ambiente, já que esse elemento é considerado essencial e é encontrado naturalmente em altas concentrações. Em contrapartida, em Wallner-Kersanach (1994), as concentrações de $\mathrm{Zn}$ da estação considerada controle variaram de 49,3 a 55,5 $\propto \mathrm{g} \mathrm{g}^{-1}$; desta forma, quando as concen- trações obtidas neste estudo são comparadas a esses valores é possível concluir que o $\mathrm{Zn}$ está contribuindo para a contaminação do organismo.

Com relação às concentrações máximas permitida pela Legislação Brasileira para contaminantes inorgânicos em pes-cados, nenhum dos metais analisados apresentou concentrações acima do limite estabelecido.

Alguns metais analisados neste estudo $(\mathrm{Cu}, \mathrm{Fe}, \mathrm{Mn}, \mathrm{Ni}$ e $\mathrm{Zn}$ ) não apresentam limites máximos recomendados para consumo humano na Legislação Brasileira. Esses metais são considerados essenciais na manutenção do metabolismo dos organismos, porém, em concentrações elevadas podem ser tóxicos (Miguel et al., 1999). A ausência de limites máximos estabelecidos pela legislação torna difícil determinar o nível de impacto desses metais na comunidade consumidora e pesqueira local.

Em relação ao índice de poluição do metal, a Fazenda Cajaíba alcançou concentrações mais críticas que as demais áreas estudadas. Dessa forma, pode-se concluir que a refinaria de petróleo não é o veiculador desse metal, pela localização da estação amostral. Essas concentrações mais elevadas de Cd, além de Fe, $\mathrm{Mn}$ e $\mathrm{Zn}$, certamente se devem à contribuição do setor norte da região, onde se encontra uma fonte potencial desses elementos, provenientes da deposição de resíduos produzidos por uma fundição primária de chumbo, no período de 1960 a 1993: uma subsidiária da multinacional Penarroya, em Santo Amaro da Purificação, BA. Esses resíduos, segundo estudos realizados em 1995, evidenciaram elevadas concen-trações de chumbo nos sedimentos e em moluscos em todo o ecossistema ao norte da BTS, onde deságua o rio Subaé, após receber uma carga poluidora da fundição (Carvalho et al., 2003).

Indústrias que processam materiais contendo $\mathrm{Cd}$ emitem esse metal na atmosfera e também liberam resíduos sólidos e efluentes líquidos contendo Cd (Wallner-Kersanach, 1994; Piotrowski \& Coleman, 1980). O Cd pode apresentar-se em pequenas concentrações em fertilizantes fosfatados (Tavares, 1996), desta forma, parece provável que a atividade de agricultura 
contribui em pequena escala para a poluição desse elemento em áreas do norte-nordeste da baía.

\section{CONCLUSÃO}

As estações amostradas, inclusive a estação de referência, apresentaram concentrações de $\mathrm{Cu}, \mathrm{Zn}$ e Fe semelhantes a áreas consideradas como moderadamente contaminadas citadas neste trabalho. Com base nos estudo de Wallner-Kersanach (1994), é possível concluir que houve enriquecimento de $\mathrm{Cd}, \mathrm{Cu}$ e $\mathrm{Zn}$ pelo molusco em questão nos últimos dez anos.

Os resultados apresentados neste trabalho apontam a necessidade de contínuo monitoramento da biota, na região norte e nordeste da Baía de Todos os Santos, utilizada como fonte de renda e para consumo próprio pela população local, a fim de que se possa dar subsídios para futuros programas de controle de poluição ambiental.

\section{REFERÊNCIAS BIBLIOGRÁFICAS}

ANVISA, 1998, Agência Nacional de Vigilância Sanitária de Alimentos DINAL. Portaria n. 685 de 27/08/1998. Diário Oficial de 24/09/98. Disponível em: http://www.anvisa.gov.br/legis/portarias/ 685_98.htm. Acesso em: 15 ago. 2005.

ARAGON, G. T., 1986, Distribuição espacial de nutrientes e metais pesados em sedimentos e águas superficiais em um ecossistema de manguezal. Acta Limnol. Brasil., 1: 365-385.

CAMUSSO, M., BALESTRINI, R. \& BINELli, A., 2001, Use of zebra mussel (Dreissena polymorpha) to assess trace metal contamination in the largest Italian subalpine lakes. Chem., 44: 263-270.

CARVALHO, C. E. V.; LACERDA, L. D. \& GOMES, M. P., 1993. Metais pesados na biota bêntica da Baía de Sepetiba e Angra dos Reis, RJ. Acta Limnol. Brasil., 6: 222-229.

Carvalho, G. P., Cavalcante, P. R. S., CAStro, A. C. L. \& ROJAS, M. O. A. I., 2001, Preliminary assessment of heavy metal levels in Mytella falcata (Bivalvia, Mytilidae) from Bacanga river estuary, São Luís, State of Maranhão, Northeastern Brazil. Rev. Brasil. Biol., 60(1): 11-16.

CARvalho, F. M., SILVANY-NETO, A. M., TAVARES, T. M., COSTA, A. C. A., CHAVES, C. R., NASCIMENTO, L. D. \& REIS, M. A., 2003, Chumbo no sangue de crianças e passivo ambiental de uma fundição de chumbo no Brasil. Rev. Panam. Salud Publica, 13(1): 19-24.

COHEN, B. L., 1985, Bioaccumulation factor in marine organism. Health Physics, 49(6): 1290-1294.

COIMBRA, G. A., 2003, Distribuição de metais pesados em moluscos e sedimento nos manguezais de Coroa Grande e da Enseada das Garças, Baía de Sepetiba, RJ. Dissertação de Mestrado, Departamento de Geociências, UFF, Niterói, 72p.

CRA (CENTRO DE RECURSOS AMBIENTAIS), 2001, Avaliação da qualidade das águas costeiras superficiais. Relatório Técnico/Avaliação Ambiental - período 2001. CRA, Salvador.

DASKALAKIS, K. D., 1996, Variability of metal concentrations in oyster tissue and implications to biomonitoring. Mar. Pollut. Bull. 32: 794801 .

FREEDMAN, B., 1995, Environmental ecology: the ecological effects of pollution, disturbance and other stresses. $2^{\text {nd }}$, Academic Press, San Diego.

FÖRSTNER, W. G. T .W., 1993, Metal pollution in the aquatic environment. $2^{\text {nd }}$, Springer Verlage, New York, 486p.
GARCIA, K. S., OLIVEIRA, O. M. C., QUEIROZ, A. F. S. \& ARGOLO, J. L., 2007, Geoquímica de sedimentos de manguezal em São Francisco do Conde e Madre de Deus - BA. Geochim. Brasil., 21(2) 167-179.

GERMEN/UFBA-NIMA, 1997, Baía de Todos os Santos. Diagnóstico socioambiental e subsídios para a gestão. Salvador, 244p.

LACERDA, L. D., 1983, Aplicação da metodologia de abordagem pelos parâmetros críticos no estudo da poluição por metais pesados na Baía de Sepetiba. Tese de Doutorado, Departamento de Biofísica, UFRJ, Rio de Janeiro, 135p.

LESSA, G. C., DOMingueZ, J. M. L., BitTencourT, A. C. S. P. \& BRICHTA, A., 2001, The tides and tidal circulation of Todos os Santos Bay, Northeast Brazil: a general characterization. An. Acad. Bras. Cienc., 73: 245-261.

LOBEL, P. B., MOGIE, P., WRIGHT D. A. \& WU, B. L., 1982, Metal accumulation in four molluscs. Mar. Pollut. Bull., 13: 170-174.

MASFARAUD, J. F., PFOHL-LESKOWICZ, A., MALAVEILlE, C. \& MONOD, G. 1992, DNA adducts formation and 7-ethoxyresorufin Odeethylase induction in primary culture of rainbow trout hepatocytes exposed to benzo [a] pyrene. Toxic. In Vitro, 6: 523-531.

MIGUEL, C. S., MACHADO, L. M. \& BEBIANNO, M. J., 1999, Concentração de $\mathrm{Cd}, \mathrm{Cu}$ e $\mathrm{Zn}$ em mexilhões Mytilus galloprovincialis e lapas Patella aspera, ao Longo da Costa Algarvia (Sul de Portugal). Ecotoxicol. and Environ. Rest., 2(1): 1-6.

MOUEZA, M., GROS, O. \& FRENKIEL, L. 1999, Embryonic, larval and postlarval development of the tropical clam, Anomalocardia brasiliana (Bivalvia, Veneridae). J. Molluscan Stud., 65: 73-88.

NRIAGU, J. O., 1990, Global metals pollution: poisoning the biosphere. Environment, 32: 7-33.

PESO-AGUIAR, M. C., 1991, Degradação Ambiental da Baía de Todos os Santos. Análise \& Dados, 1: 55-57.

PHILLIPS, D. J. H. \& SEGAR, D. A., 1986, Use of bio-indicators in monitoring conservative contaminants: program design imperatives. Mar. Pollut. Bull., 17: 10-17.

PHILliPS, D. J. H., ThOMPSON, G. B., GABUJ, K. M. \& HO, C. T., 1982, Trace metals of toxicological significance to man in Hong Kong seafood. Environ. Pollut. Ser. B. 3: 27- 45.

PIOTROWSKI, J. K. \& COLEMAN, D. O., 1980, Environmental hazards of heavy metals: summary evaluation of lead, cadmium and mercury. General Report, Monit. and Assess. Res. Center, 20: 42.

REZENDE, C. E. \& LACERDA, L. D., 1986, Metais pesados em mexilhões (Perna perna) no litoral do Estado do Rio de Janeiro. Rev. Brasil. Biol., 46: 239-247.

SANTOS, J. B., 2002, Estudos geoquímicos em substrato lamoso em zonas de manguezal da região de São Francisco do Conde - Recôn-cavo Baiano: subsídios a um programa de diagnóstico e monito-ramento ambiental para regiões de manguezal influenciadas por atividades petroliferas. Dissertação de Mestrado, Instituto de Geo-ciências, Universidade Federal da Bahia, 142p.

SOUZA, S. P., TAVARES, T. M. \& BRANDÃO, A. M., 1978, Distribuição de chumbo, cádmio e zinco em sedimentos no estuário do Rio Subaé, Bahia. Supl. Cien. Cult., 30: 406.

SZEFER, P., GELDON, J., ALI, A. A., BAWAZIR, A. \& SAD, M., 1997, Distribution and association of trace metals in soft tissue and byssus of of mollusc Perna perna from the gulf of Aden, Yemen. Environment Inter. 1(23): 53-61.

TAVARES, T. M. \& PESO, M. C., 1979, Avaliação dos riscos de intoxicação por mercúrio através de ingestão de bivalves comestíveis, da enseada dos Tainheiros. Cien. Cult. Supl., 31: 434.

TAVARES, T. M., 1996, Programa de Monitoramento dos Ecossistemas ao norte da Baía de Todos os Santos. Relatório Técnico Final. Universidade Federal da Bahia, Salvador.

THOMPSON, M.-F., SANCHEZ, G. M., VIRENDER, K. S., 1987, Trace metals in the oyster Crassostrea virginica of the Terminos Lagoon, Campeche, México. Mar. Pollut. Bull., 26(7): 41-53. 
USERO, J., GONZALEZ-REGALADO, E. \& GARCIA, I., 1997, Trace metals in the bivalve molluscs Ruditapes decussate sand Ruditapes philippinarum from the Atlantic Coast of Southern Spain. Environ. Int., 23(3): 291-298.

WALLNER-KERSANACH, M., 1994, Assessment of trace metal polution of Todos os Santos bay on the basis of measurements of bivalves and sediments. PhD Thesis, Universität Bremen, Bremerhaven, 114p.
WALLNER-KERSANACH, M., LOBO, S. E. \& DA SILVA, E. M., 1994, Depuration effects on trace metals in Anomalocardia brasiliana (Gmelin, 1791). Bull. of Environ. Contam. and Toxicol., 52: 840-847.

ZAR, J. H., 1996, Biostatistical analysis. $3^{\text {rd }}$ ed., Prentice-Hall, London, $662 \mathrm{p}$. 